\title{
Efficient Multipath Routing and Wavelength Allocation Using Traffic Grooming in Optical Wavelength Division Multiplexing Mesh Networks
}

\author{
${ }^{1}$ Kavitha, T. and ${ }^{2}$ V. Rajamani \\ ${ }^{1}$ Department of Electronics and Communication Engineering, \\ NPR College of Engineering and Technology, Natham, Dindigul, Tamilnadu, India \\ ${ }^{2}$ Department of Electronics and Communication Engineering, \\ Indra Ganesan College of Engineering, Manikandam, Tiruchirappalli, Tamilnadu, India
}

Received 2012-05-28, Revised 2012-09-05; Accepted 2012-09-08

\begin{abstract}
In optical WDM mesh networks, traffic grooming is essential in order to utilize the bandwidth efficiently to minimize the cost and meet the user service requirement. By grooming traffic, it is possible to reduce the total cost of all Add drop multiplexer needed. Also for the high processing capability in a WDM network, the path should be capable of carrying the traffic without any failure. In this study, we use a traffic grooming algorithm to establish light paths between node pairs having the largest amounts of traffic. Among the established light paths, the risk-disjoint paths are obtained using the large traffic first algorithm. After establishing light paths, the traffic is routed from the source to the connection node and then traffic is transmitted from the connection node to the destination node. In addition to the efficient routing, we propose a wavelength allocation technique to ensure that the traffic is transferred to the destination with efficient use of the network resources. From the simulation results we show that this algorithm involves additional capacity in the virtual topology to support survivable routing of connections.
\end{abstract}

Keywords: WDM Networks, Traffic Grooming, Wavelength Allocation, Routing, Establishing Light Paths, Survivable Routing, Network Resources, Risk-Disjoint Paths

\section{INTRODUCTION}

\subsection{WDM Networks}

Wavelength Division Multiplexing (WDM) provides huge amount of optical bandwidth for telecom operators to satisfy the increasing traffic demands and provide high speed connectivity for achieving faster transfer rate for the high speed communication. In WDM network, the optical transmission is thus divided into number of non overlapping wavelength bands where each of the wavelengths supports a particular single communication channel. WDM is further classified into two broad categories: (1) Coarse WDM with nearly 40 wavelengths per channel and (2) Dense WDM with nearly 160 wavelengths per fiber where each of the wavelengths can be viewed as a channel that provides optical connectivity between two nodes. WDM channels co- exist on single fiber where we can tap into huge fiber bandwidth with corresponding challenges of design and development of most appropriate network architecture, protocol and algorithms.

Each fiber can carry a large number of high-capacity channels. Dividing these channels into lower-bandwidth virtual connections between couples of nodes gives rise to technical difficulties: wavelengths are at most a few hundreds; therefore they need to be time-multiplexed to be shared among many couples of communicating nodes. The key components to achieve time multiplexing, the Add-Drop Multiplexers (ADM), are needed each time a wavelength has to be processed electronically at a node in order to add or drop packets. Therefore they represent a significant fraction of the total cost of the infrastructure (Sinha et al., 2012). Corresponding Author: Kavitha, T., Department of Electronics and Communication Engineering, NPR College of Engg. And Tech., Natham,
Dindigul, Tamilnadu, India 


\subsection{Traffic Grooming}

Traffic grooming is defined as the intelligent allocation of the demands onto available wavelengths such that the cost of the network is minimized. In Wavelength Division Multiplexing (WDM)-based networks the bandwidth of a wavelength channel is high when compared with the bandwidth of connection requests from higher layers, such as the Internet Protocol (IP) layer. In this case traffic grooming can lead to an efficient use of wavelength channel bandwidth. Traffic grooming describes how low speed connections are packed into high capacity connections, such as lightpaths, avoiding the waste of bandwidth that rises from the mismatch between a connection demand and the lightpath capacity. Traffic grooming is a key issue in optical WDM networks, which must be cost-effective (Patel et al., 2010).

Traffic grooming improves resource utilization for both unicast and multicast traffic. It consists of multiplexing traffic (usually statistically) from different demands in the same lightpath or light-tree, as well as to route one demand through several lightpaths and/or light-trees. Routing unicast and multicast traffic together in a network represents a challenge taking into account the limitations of the WDM technology. Traditionally, there are two schemes for performing this: (i) light-trees groom together unicast with multicast traffic; thus OEO conversions are reduced but, unicast traffic can arrive to undesired nodes (incurring in bandwidth wastage) and (ii) unicast traffic is routed using lightpaths through different wavelengths than those used by light-trees (Paul and Shrivastava, 2010).

In optical networks employing Wavelength Division Multiplexing (WDM) technology, the capacity of a fiber is divided into several non-overlapping wavelength channels that can transport data independently. These wavelength channels make up lightpaths, which are used to establish optical connections that may span several fiber links. With current commercial technology, each lightpath can be independently operated at a data rate ranging up to $100 \mathrm{~Gb} / \mathrm{s}$. However, traffic between a pair of nodes may not be able to fill up the available bandwidth of a lightpath. In order to efficiently utilize the available bandwidth, several independent traffic streams can be aggregated to share the capacity of a lightpath. This is known as traffic grooming (Chen et al., 2010).

\subsection{Need for Traffic Grooming in WDM Networks}

In an optical WDM Network, every node may have one SONET Add/Drop Multiplexer (ADM) for each wavelength. Instead, with traffic grooming it may be possible to have ADM for the wavelength used at that node; other wavelengths may be optically routed without electronic switching. To efficient utilize the bandwidth in a WDM system, traffic grooming can be used to combine lower rate traffic stream on to available wavelengths in order to minimize the cost and meet the user service requirement (Paul and Shrivastava, 2010).

In order to save network cost and to improve network performance, it is very important for the network operator to be able to "groom" the multiple lowspeed traffic connections onto high-capacity circuit pipes (Chen et al., 2010).

By intelligently grooming traffic, we can reduce the total cost of all Add drop multiplexer ADMs needed. The potential saving can be significant and grows with both the network size and inter nodal demand (Sinha et al., 2012).

The light-paths are assumed to be full-duplex and the forward and reverse direction signals use the same wavelength and path. Unless a wavelength carries traffic destined for a given node or needs regeneration, it passes through optically. Otherwise, the following take place: (1) the lightpath is terminated, (2) the traffic is processed electronically (and regenerated simultaneously), (3) traffic destined to the node is dropped and (4) the rest of the traffic, including locally added traffic, if any, is forwarded on other lightpaths through the transceivers. Thus traffic grooming is required (Beshir et al., 2011).

In Optical-Electrical-Optical (OEO) conversions are usually avoided by network operators because more network resources (e.g., transmitters and receivers) are needed to regenerate optical signals. Moreover, OEO conversions could delay traffic since traffic flowing must be processed and queued electronically. Therefore, the way in which traffic is groomed affects not only the performance of the network but also its throughput (Ramesh and Vadivelu, 2010).

\subsection{Previous Work}

In our previous paper (efficient multipath routing and wavelength allocation for optical WDM mesh networks), we have designed an efficient IP router for the WDM network in order to satisfy the optical constraints. We design a router for data transmission. The multiple paths toward each destination are set from the source node. Multiple routes can be discovered for data transmission using the router and the paths which satisfy the optical constraints are set for data transmission. The traffic routing is performed by choosing the connection node.

Along with routing, we also propose to allocate wavelength to every path such that complete bandwidth is effectively utilized. We have proposed a technique in which wavelength allocation is performed before transmission of the data in order to make the system efficient in terms of wavelength.

As an extension to this study, we use a traffic grooming algorithm in order to enhance the traffic. This algorithm tries to establish light paths between node 
pairs having the largest amounts of traffic. Depending upon the resources to be shared among the backup paths, the protection schemes are divided into dedicated protection and shared protection. We use two times Dijkstra's risk-disjoint path algorithm for dedicated protection in order to establish two lightpaths. For a shared protection, we use dijkstra algorithm in order to establish a lightpath. By this algorithm, additional capacity can be involved in the virtual topology to support survivable routing of connections.

\subsection{Related Work}

Huang and Mukherjee (2010) have proposed and investigate the characteristics of survivable multipath traffic grooming with connection-level protection in telecom mesh networks.

Their results indicate that (1) the network performance can be notably improved by exploiting the inversemultiplexing capability, (2) tight constraints have negative impact on performance and (3) use of a single primary path after multipath failure is helpful to reduce the impact.

Ab-Rahman (2012) developed a combined approach of Optical Add-Drop Multiplexer (OADM) and Optical Cross Connect (OXC). Their technique enhances increase the survivability in the ring network. With the 'Accumulation' feature and ' $U$ ' turn reflection enable the multiplex, linear and ring protection scheme activated to ensure the signal arrives at the customer although breakdown occurs in the line and optical node. They also performed topology migration without restructuring process due to randomly growth of new nodes.

Saleh and Kamal (2009) have addressed the problem in both non-splitting networks where the nodes do not have optical splitting capabilities and in splitting networks where the nodes do have optical splitting capabilities. First, we formulate the problem in each of the two networks as a Mixed Integer Linear Programs (MILP). Afterwards, based on observations from optimal solutions, we develop a heuristic approach for each network by relaxing and simplifying its corresponding MILP.

Ramesh et al. (2011) have proposed Distributed dynamic traffic grooming RWA algorithm (DTMR). Here optimal primary, secondary and backup paths are selected. The path selection is based upon minimal blocking probability. This algorithm is proactive, in the sense that we are minimizing blocking probability, maximizing the throughput and the number of requests processed at the source node at a given time. This approach is self regulating, it automatically adapts to various traffic conditions across the network.

Huang et al. (2010), have investigated the characteristics of survivable multipath traffic grooming with respect to typical constraints. Their grooming technqiue significantly outperforms single-path traffic grooming. Both typical constraints (DDC and IMF) impact the performance in the metrics of bandwidthblocking ratio and resource overbuild.

\section{MATERIALS AND METHODS}

\subsection{Proposed Work}

In WDM networks, traffic grooming can be done efficiently by utilizing the bandwidth. Here we use Two Times Disjoint-Risk Disjoint Path (TTD-RDJP) algorithm for solving the two Survivable Traffic Grooming (STG) problems.

The two STG problems include:

- Sub-Wavelength Connection Survivable Routing (SWCSR) problem and

- Protection Aware Virtual Topology Design (PAVTD) problem

Large Traffic First-(LTF-SWCSR) algorithm is used for SWCSR problem which uses TTD-RDJP algorithm to find the risk-disjoint paths in the virtual topology for connections.

Maximizing Single-Hop Traffic (MSHT-PAVTD) algorithm is used for PAVTD problem which uses TTDRDJP algorithm to find risk-disjoint paths in the physical topology for light-paths.

We consider protection schemes in this work. Protection schemes are divided into dedicated protection and shared protection schemes depending on whether resources can be shared among backup paths or not.

- In dedicated protection, traffic is transmitted simultaneously over two disjoint paths and a switch is used at the receiving node to choose the better signal

- In shared protection, the traffic is transmitted over the primary path at normal operation time and the backup paths can share common resources only if their primary paths are node (or link) disjoint. Shared protection offers better resource utilization at the cost of a longer recovery time

\subsection{Two Times Disjoint-Risk Disjoint Path (TTD-RDJP) Algorithm}

TTD-RDJP is an adaptive algorithm in that it updates the link weights of the network according to the current network state. Equation 1 defines the link weight function $\mathrm{L}(\mathrm{a}, \mathrm{b})$ for a light-path $(\mathrm{a}, \mathrm{b}) \mathrm{L}$. $(\mathrm{a}, \mathrm{b})$ is used as a light-path metric while searching for primary paths of connections:

if $L(a, b)=\left\{\sum_{(p, q) \in(a, b)} k(p, q) \eta_{f}(a, b) \geq \eta_{r}^{\infty}\right\}$ Otherwise

where, $\eta_{\mathrm{r}}$ is the bandwidth requirement of the connection, $\eta_{\mathrm{f}}(\mathrm{a}, \mathrm{b})$ is the free bandwidth on the lightpath $(a, b)$. 
When shared protection scheme is used, backup connections can share bandwidth within a lightpath if their primary connections are risk-disjoint. Generally, we can evenly divide the bandwidth of a lightpath into $\mathrm{S}$ channels. Each channel has a bandwidth equal to the smallest grooming granularity. Depending on the usage, the channels can be classified into three categories, namely dedicated, spare and free channels. A channel is dedicated if it is assigned to a primary connection. A channel is a spare channel if it is assigned to a backup connection. The channels not assigned to any connections are free channels. When a primary connection $\mathrm{n}$ is specified, a spare channel $\mathrm{s}$ can be further classified as a sharable spare channel or a nonsharable spare channel depending on whether $\mathrm{n}$ is riskdisjoint with all the primary connections whose backup connections share the spare channel $\mathrm{s}$. If they are risk disjoint, then $\mathrm{s}$ is sharable to $\mathrm{n}$; otherwise, $\mathrm{s}$ is not sharable to $\mathrm{n}$. Note that for the dedicated protection scheme, all spare channels are not sharable.

To fully exploit the backup bandwidth sharing to reduce spare capacity consumption, a different link weight function is defined for lightpaths while searching for backup paths of connections. As shown in (2), the backup lightpath weight $\mathrm{Bl}(\mathrm{a}, \mathrm{b}, \mathrm{n})$ also depends on the primary connection $\mathrm{n}$. With (2), the grooming algorithm tries to assign as many sharable spare channels to a backup connection as possible. Only if the amount of sharable spare channels is not enough, does it assign free channels to the backup connection Equation 2:

$$
\begin{aligned}
& \left\{\psi \times \sum_{(\mathrm{m}, \mathrm{n}) \in(\mathrm{a}, \mathrm{b})} \mathrm{k}(\mathrm{p}, \mathrm{q})\right. \\
& \operatorname{Bl}(\mathrm{a}, \mathrm{b}, \mathrm{n})=\left\{\left(\theta+\psi(1+\theta) \sum_{(\mathrm{m}, \mathrm{n}) \in(\mathrm{a}, \mathrm{b})} \mathrm{k}(\mathrm{p}, \mathrm{q}) \infty\right\}\right. \\
& \text { if } \eta_{\mathrm{s}}(\mathrm{a}, \mathrm{b}, \mathrm{n}) \geq \eta_{\mathrm{r}} \\
& \text { if } \eta_{\mathrm{s}}(\mathrm{a}, \mathrm{b}, \mathrm{n})+\eta_{\mathrm{f}}(\mathrm{a}, \mathrm{b}) \geq \eta_{\mathrm{r}}^{\text {and }} \eta_{\mathrm{s}}(\mathrm{a}, \mathrm{b}, \mathrm{n})<\eta_{\mathrm{r}} \\
& \text { otherwise }
\end{aligned}
$$

where, $\eta_{s}(a, b, n)$ is the amount of sharable spare bandwidth on the light path $(a, b)$ with respect to the primary connection $n, \theta=1-\eta \mathrm{s}(\mathrm{a}, \mathrm{b}, \mathrm{n}) / \eta \mathrm{r}$ is the ratio of the newly reserved bandwidth $\left(\eta_{\mathrm{r}}-\eta_{\mathrm{s}}(\mathrm{a}, \mathrm{b}, \mathrm{n})\right)$ to the bandwidth requirement $\left(\eta_{\mathrm{r}}\right), \psi(0<\psi<1)$ is a parameter to weight sharable bandwidth. By making $\psi$ a number smaller than 1, we encourage the grooming algorithm to choose paths using sharable channels instead of free channels. On the other hand, $\psi$ should not be set too small to avoid using too many sharable channels unnecessarily. Note that for dedicated protection, $\eta \mathrm{s}(\mathrm{a}, \mathrm{b}, \mathrm{n}) \equiv 0$ regardless of the primary connection $n$, because all spare bandwidths are not sharable.

The Algorithm 1 given below explains TTD-RDJP:

Algorithm 1
1. Update the link weights of network $\mathrm{N}$ according to the current state of the network.

2. Run Dijkstra algorithm to select shortest path.

3. If shortest path is the primary path

Delete any link in $\mathrm{N}$ that shares at least one risk with any link in primary path. End if

4. For shared protection, the link weights of $\mathrm{N}$ are updated after the link is deleted.

5. Repeat step 2 on network $\mathrm{N}$ to select another shortest path for backup path.

\subsection{Maximizing Single-Hop Traffic (MSHT- PAVTD) algorithm}

We propose the maximizing Single-Hop Traffic (MSHT-PAVTD) heuristic grooming algorithm which tries to establish light-paths between node pairs having the largest amounts of traffic. In MSHT-PAVTD, TTDRDJP is used to find two risk-disjoint paths and Dijkstra's algorithm is used to find the shortest path.

Initially the connection requests are set to form a traffic matrix. All the traffic matrices are gathered to form a single residual traffic matrix. Then the node pair with maximum residual bandwidth is selected to establish a light-path on risk-disjoint paths.

Algorithm 2 given below explains the MSHT-PAVTD.

\section{Algorithm 2}

1. Let traffic matrix be $\mathrm{T}$ and the sum of all traffic matrices be ST. The total bandwidth needed from source node to destination be $\operatorname{ST}(\mathrm{s}, \mathrm{d})$.

2. $\mathrm{ST}(\mathrm{s}, \mathrm{d})=\sum_{\mathrm{x} \in \mathrm{X}}\left(\mathrm{S}_{\mathrm{T}}^{\mathrm{x}}(\mathrm{s}, \mathrm{d}) \times \mathrm{x}\right)$, where $\mathrm{X}$ is the set of low-speed connection granularities.

3. If an element of $\mathrm{T} \neq 0$

Select node pair with maximum residual bandwidth in $\mathrm{T}$.

\section{End if}

4. If dedicated protection is concerned.

Establish two light-paths from source to

destination on risk disjoint paths $\mathrm{A}$ and $\mathrm{B}$ using algorithm 1.

Else if Shared protection is concerned

Establish a light-path from source to destination on path A using Dijkstra algorithm

End if

In this Fig. 1, node $m$ is the source node and node $n$ is the destination node. For a dedicated protection, the source establishes two lightpaths (m-a-e-h-l-n) and (m-b$\mathrm{f}-\mathrm{k}-\mathrm{n})$. According to TTD-RDJP the path A acts as a primary path and during failure of primary path, path $B$ is chosen for traffic transmission. 


\subsection{Large Traffic First (LTF-SWCSR) Algorithm}

Large Traffic First Algorithm (LTF-SWCSR) is used to find the risk-disjoint paths in the virtual topology for connections. When many connection requests enter the network, the connection requests are sorted in a nonincreasing traffic amount order. The connections are established from source to destination on one path and other path reserves the bandwidth.

\section{Algorithm 3}

1. Sort the connection requests in a list $\mathrm{L}$ in nonincreasing traffic amount order.

2. When list L is not empty, acquire and remove the connection request from head of $\mathrm{L}$.

3. If dedicated protection is concerned

3.1 Establish primary connection on path $\mathrm{A}$ and backup connection on path $B$.

else if shared protection is concerned

3.2 Establish path $\mathrm{C}$ for connection between source and destination.

3.3 Reserve Bandwidth on path D

End if

4 Use algorithm 4 for transmission of traffic

End if (Wang and Ramamurthy, 2004)

In this Fig. 2, the source node $m$ establishes primary connection (m-a-e-h-l-n) and backup connection (m-b-f$\mathrm{k}-\mathrm{n}$ ) to the destination $\mathrm{n}$. According to the shared protection path $\mathrm{C}$ (m-c-g-i-n) is established for connection between source and destination and path $\mathrm{D}$ (m-d-j-o-n) is established for bandwidth reservation.

\subsection{Transmission Using Connection Node}

We propose a technique in which the router placed at the source transfers the data to the connection node and from the connection node, the data is sent through the multiple paths towards the destination with wavelength allocation.

In phase 1 , the traffic is routed from the source to the connection node by the router through the link disjoint path and in phase 2 the traffic is transmitted from the connection node to the destination through the link disjoint path set.

In Fig. 3, the source node is initially connected with the node $b$. Due to congestion at the node $b$ along the path the router sends a warning message (indicated by red arrow) to the source $\mathrm{m}$. Then source node sends connection request to the neighbors of node $b$ (i.e.,) node $\mathrm{a}$ and $\mathrm{b}$ (indicated by blue arrow). According to the load traffic, the node a is selected as the connection node. Then the router establishes link disjoint path set from source node to connection node a. The router searches multiple paths from node a to node $\mathrm{n}$ for congestion control (indicated by black arrows).
Um: Link Disjoint Path from source node $\mathrm{m}$ to connection node a

Vm: Link Disjoint Path from source node $\mathrm{m}$ to node $\mathrm{c}$

Ym: Link Disjoint Path set from connection node a to destination $\mathrm{n}$

Yt: Traffic associated with path set Ym

Ut: Source node traffic

Tq: Traffic request

Cr: Connection from source $\mathrm{m}$ to node $\mathrm{b}$

Cw: Congestion warning

\subsection{Phase 1}

When the transmission is not efficient due to insufficient channel availability or congestion at a specific node along the path, then a warning message is sent to the router by the node.

If the routing of the traffic from source $m$ requires $j$ channels and if the maximum channel capacity of the node $b$ is $i$ then:

- $\quad$ if $\mathrm{j}<\mathrm{i}$; then there is sufficient channel availability and this node is selected for traffic

- routing

- if $\mathrm{j}>\mathrm{i}$; then the channel availability is insufficient and congestion occurs at node $b$ so

It sends a congestion warning message, $\mathrm{Cw}$ to the router as shown in Fig. 1.

The router calculates the overloaded traffic at the congested node as:

Overloaded traffic $=$ total

traffic-traffic handled $t_{L}=U_{t} U_{h}$

The router then sends a traffic request, $\mathrm{Tq}$ to the neighbor nodes; $a$ and $c$ of the congested node $b$ using the routing table information in the router as shown in Fig. 1.

As a return to the traffic request $\mathrm{Tq}$, the router receives information of the neighbor nodes which consist of node id, current load and channel capacity.

The total load on each node is the sum of the loads of the paths passing through that node.

Load on node $a, a_{L}$ and load on node $c, C_{L}$ is calculated as:

$A_{L}=$ load on path Um

$\mathrm{C}_{\mathrm{L}}=$ load on path $\mathrm{Vm}$

The router then checks the channel availability for the overloaded traffic in each of the neighbor nodes. If the overloaded traffic is $t_{L}$ then:

- if $\mathrm{t}_{\mathrm{L}}<\mathrm{C}_{\mathrm{L}}$; then node $\mathrm{c}$ does not get overloaded and can be used as the connection node

- if $t_{L}>C_{L}$; then node $c$ is overloaded and hence cannot be used as the connection node 


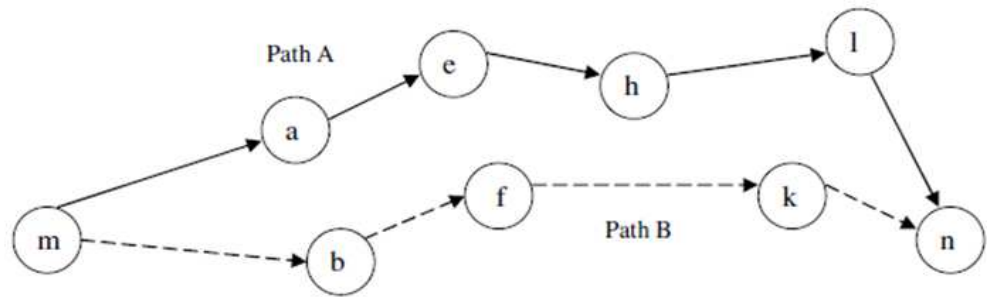

Source

(c)
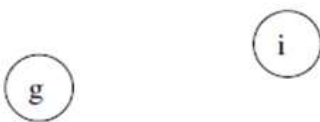

Destination
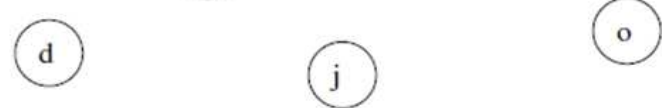

Fig. 1. Establishment of lightpaths in MSHT-PAVTD
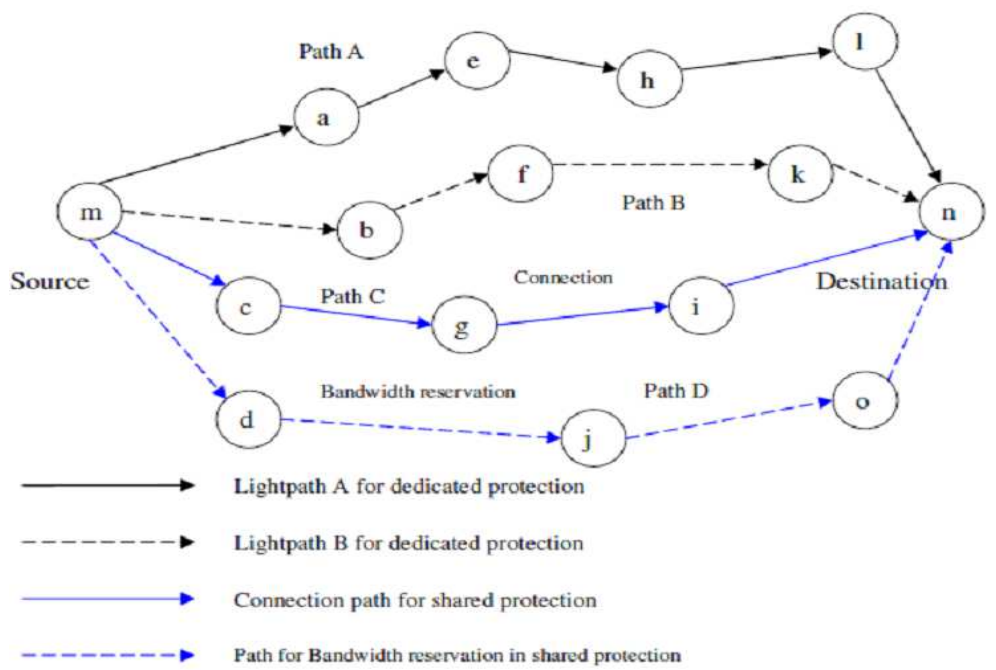

Fig. 2. Establishment of lightpaths in LTF-SWCSR

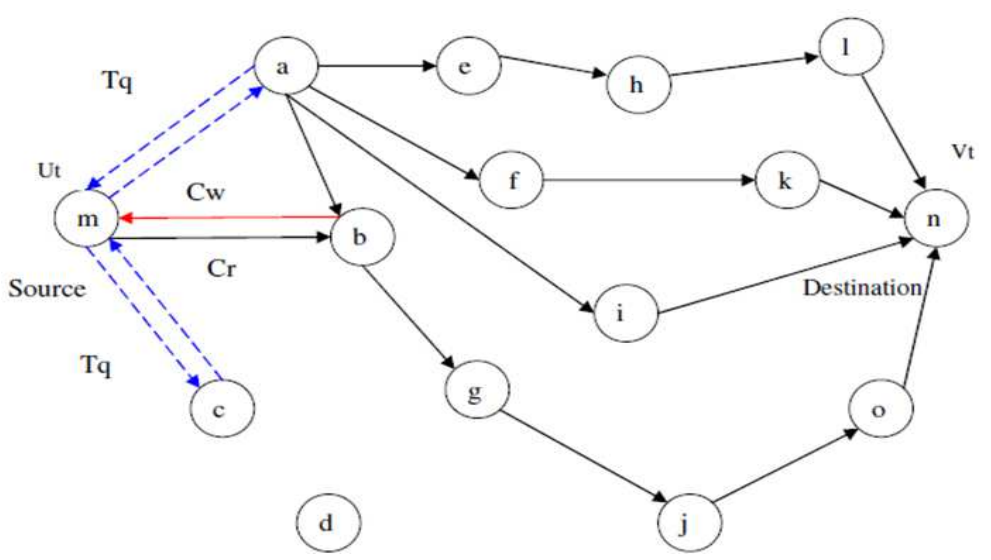

Fig. 3. Determination of connection node and establishment of multiple paths 
If the neighbor node $\mathrm{c}$ is fully loaded, then it cannot be selected as a connection node and so the router will start enquiring about other neighboring node; node a. If is the load on neighbor node a then:

- $\quad$ if $\mathrm{t}_{\mathrm{L}}<\mathrm{a}_{\mathrm{L}}$; then node a does not get overloaded and can be used as the connection node

- if $t_{L}>a_{L}$; then node a is overloaded and hence cannot be used as the connection node

In this way, one of neighboring nodes is selected as the connection node. If node a has sufficient capacity, then node a is selected as the connection node.

The router establishes a Link Disjoint Path set (LDP), Um from source node $\mathrm{m}$ to the connection node a. (using algorithms 2 and 3 ).

For a unit traffic split ratio for connection node a, the traffic associated with path Um is Ut. In the first phase, the router calculates the bandwidth per unit split ratio $\mathrm{Sr}_{\mathrm{v}}$ for connection node a across link e, as:

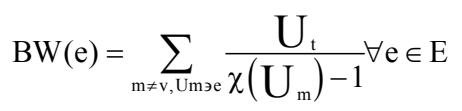

where, $x\left(U_{m}\right)$ is the number of link disjoint path in $U_{m}$.

\subsection{Phase 2}

For the congestion control, the router searches for multiple paths from a to $\mathrm{n}$ which are least loaded. The router establishes the link-disjoint path set $Y_{m}$ from a to $n$.

For a traffic split ratio of 1 for connection node a, the traffic associated with path set $Y_{m}$ is $Y_{t}$.

The router calculates the bandwidth per unit split ratio $\mathrm{Sr}_{\mathrm{v}}$, for the second phase as:

$$
\mathrm{BW}(\mathrm{e})=\sum_{\mathrm{n} \neq \mathrm{v}, \mathrm{Y}_{\mathrm{n}} \mathrm{e}} \frac{\mathrm{Y}_{\mathrm{t}}}{\chi\left(\mathrm{Y}_{\mathrm{m}}\right)-1} \forall \mathrm{e} \in \mathrm{E}
$$

where, $\mathrm{x}\left(\mathrm{Y}_{\mathrm{m}}\right)$ is the number of link disjoint path in $\mathrm{Y}_{\mathrm{m}}$.

After phase 2, the router computes the bandwidth, BW per unit split ratio $\mathrm{Sr}_{\mathrm{v}}$ for intermediate node a throughout the network path as:

$$
\mathrm{BW}(\mathrm{e})=\sum_{\mathrm{m} \neq \mathrm{v}, \mathrm{Um} \text { əe }} \frac{\mathrm{U}_{\mathrm{t}}}{\chi\left(\mathrm{U}_{\mathrm{m}}\right)-1}+\sum_{\mathrm{n} \neq \mathrm{v}, \mathrm{Y}_{\mathrm{n}} \text { ее }} \frac{\mathrm{Y}_{\mathrm{t}}}{\chi\left(\mathrm{Y}_{\mathrm{m}}\right)-1} \forall \mathrm{e} \in \mathrm{E}
$$

Then the router computes the maximum value $\mathrm{Sr}$ for the traffic split ratio for intermediate node, a that does not lead to violation of (original) link capacity constraints for the above required bandwidth as:

$$
\mathrm{Sr}=\min _{\mathrm{e} \in \mathrm{E}} \frac{\eta_{\mathrm{e}}}{\mathrm{BW}(\mathrm{e})}
$$

where, $\eta_{\mathrm{e}}$ is the maximum capacity constraint

For this value $\mathrm{Sr}$ of the traffic split ratio for intermediate node a, the router sends SrUt amount of traffic from node $\mathrm{m}$ to node a along path set $\mathrm{Um}$ and SrYt amount of traffic has to be transmitted from node a to node $\mathrm{n}$ along path set $\mathrm{Ym}$.

\subsection{Wavelength Allocation}

For the allocation of the efficient wavelength in the paths in the mesh network, we have selected the cost function as the main criteria.

Initially the cost function $\mathrm{q}_{\mathrm{k}}$, which is the cost of assigning wavelength $\lambda_{\mathrm{k}}$ is calculated. Based on the calculated cost function value, the wavelength is allocated in the path, from the connection node to the destination.

All the feasible wavelength in the network is given as a set $\eta=\left\{\lambda_{1}, \lambda_{2}, \ldots, \lambda_{w}\right\}$ and the cost assigned for every $\lambda_{\mathrm{k}} \in \eta$ is indicated as a set, $\mathrm{q}=\left\{\mathrm{q}_{1}, \mathrm{q}_{2}, \ldots \mathrm{q}_{\mathrm{w}}\right\}$. For each $\lambda_{\mathrm{k}} \in \eta$.

A connected component $\mathrm{G}_{\mathrm{k}}=\left\{\mathrm{V}_{\mathrm{k}}, \mathrm{E}_{\mathrm{k}}\right\}$ is first derived from the original graph, $G=\{\mathrm{V}, \mathrm{E}\}$ starting from the connection node, $\mathrm{s}$ such that if $\lambda_{\mathrm{k}}$ is available in the link leading to the destination.

The cost function, $\mathrm{q}_{\mathrm{k}}$ is calculated based on the number of edges involved in every path of graph $\mathrm{G}_{\mathrm{k}}$.

$$
\mathrm{q}_{\mathrm{K}}=\left|\mathrm{E}_{\mathrm{k}}\right|
$$

End For.

Figure 4 shows the network with the subgraph $\mathrm{G}_{\mathrm{k}}=$ $\left\{\mathrm{V}_{\mathrm{k}}, \mathrm{E}_{\mathrm{k}}\right\}$. The subgraph includes the path from the connection node to the destination. The Figure highlights the subgraph $G_{1}$ with corresponding vertices, $V_{1}$ and edges, $E_{1}$. The vertex belonging to a particular subgraph is represented by:

$$
\mathrm{V}_{\mathrm{k}}=\left\{\mathrm{V}_{\mathrm{l}}, \mathrm{V}_{2}, \ldots, \mathrm{V}_{\mathrm{n}}\right\}=\sum_{\mathrm{i}=1}^{\mathrm{n}} \mathrm{V}_{\mathrm{i}}
$$

The set of wavelengths that can be used to reach the destination is determined from the graph, $G_{k}$. The subgraph indicates the entire wavelength supported by it.

The vertices in the subgraph may support many wavelengths $\mathrm{w}\left(\mathrm{v}_{\mathrm{i}}\right)$. But only one particular wavelength for each path will be selected, based on its efficiency.

$$
\text { For each vi } \in \mathrm{V}_{\mathrm{k}} \text { : }
$$

- $\quad$ Find the cost ratio $=\mathrm{q}_{\mathrm{i}} / \mathrm{SLi}$, where $\mathrm{SLi}$ is the number of links sharing the same wavelength $\lambda_{\mathrm{k}}$

- Choose a wavelength $\lambda_{\mathrm{k}}$ from $\mathrm{w}\left(\mathrm{V}_{\mathrm{i}}\right)$ to cover $\mathrm{V}_{\mathrm{i}}$, where $\lambda_{\mathrm{k}}$ has the minimum cost ratio in $\mathrm{w}\left(\mathrm{V}_{\mathrm{i}}\right)$

- The selected wavelength is allocated to that path end for 


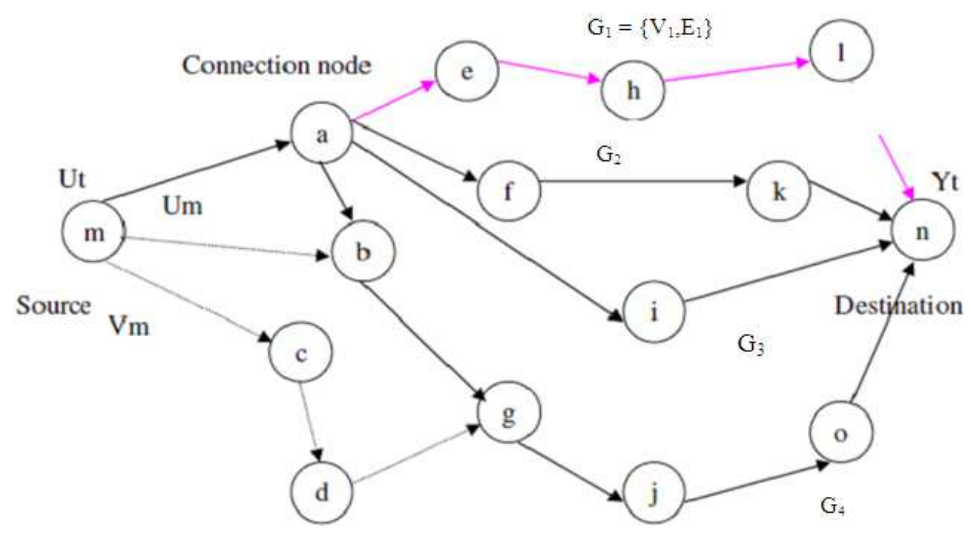

Fig. 4. Subgraph for wavelength allocation

Table 1. Simulation parameters

\begin{tabular}{ll}
\hline Topology & Mesh \\
\hline Total no. of nodes & 15 \\
Link wavelength number & $4,6,8,10$ and 12 \\
Link delay & $10 \mathrm{~ms}$ \\
Link bandwidth & $7 \mathrm{Mb}$ \\
Wavelength conversion factor & 1 \\
Wavelength conversion distance & 8 \\
Wavelength conversion time & 0.024 \\
Link utilization sample interval & 0.5 \\
Traffic arrival rate & 0.5 \\
Traffic holding time & 0.2 \\
Packet size & 200 \\
Traffic type & Exponential \\
No. of session-traffics & 4 \\
Max requests number & 50 \\
Traffic number & $1,2,3$ and 4 \\
\hline
\end{tabular}

Then the router computes the bandwidth usage $\tau(\mathrm{e})$ on link e as:

$$
\tau(\mathrm{e})=\operatorname{SrBW}(\mathrm{e}) \forall \mathrm{e} \in \mathrm{E}
$$
follows:

Now the router updates the weights $\mathrm{Wg}(\mathrm{e})$ as

$$
\mathrm{Wg}(\mathrm{e}) \leftarrow \mathrm{Wg}(\mathrm{e})\left(1+\frac{\varepsilon \tau(\mathrm{e})}{\eta_{\mathrm{e}}}\right) \forall \mathrm{e} \in \mathrm{E}
$$

Increment the split ratio $\mathrm{Sr}_{\mathrm{v}}$ associated with node a by $\mathrm{Sr}$.

Now the egress router collects all the traffic from the link disjoint path sets and then it cross checks the received traffic with the information in its routing table. If the received traffic and the routing table information coincide then the egress router transmits it towards the corresponding destination node.

\section{RESULTS AND DISCUSSION}

\subsection{Simulation Results 3.2. Simulation Model and Parameters}

We examine the performance of our Efficient Multipath Routing and Wavelength Allocation Using Traffic Grooming (MRWATG) technique with an extensive simulation study based upon the ns-2 network simulator. We use the Optical WDM network simulator (OWNs) patch in ns2. In our simulation, we simulate a 15-Node topology (as given in Fig. 1) which can be extended to any number of nodes. Various simulation parameters are given in Table 1.

In our experiments, we use a dynamic traffic model in which connection requests arrive at the network according to an exponential process with an arrival rate $r$ (call/seconds). The session holding time is exponentially distributed with mean holding time $\mathrm{s}(\mathrm{sec})$. The connection requests are distributed randomly on all the network nodes.

In all the experiments, we compare the results of MRWATG with the existing "New Technique for Routing and Wavelength Assignment (NTRWA) technique for WDM mesh networks (Chaiwong et al., 2008). In the network can be preceded by accomplishing two processes: one is searching for the shortest main route and the second shortest alternative route and another is randomly choosing wavelength.

\subsection{Performance Metrics}

We measure the following metrics in all the simulation experiments:

- Throughput in terms of packets received

- Channel utilization

- Blocking probability 


\subsection{Based on Number of Traffic Flows}

We classify the traffic flows as two types low load traffic with a load of $2 \mathrm{Mb}$ and high load traffic with a load of $8 \mathrm{Mb}$.

In the initial experiment, we vary the number of high load traffic flows as $1,2 \ldots .4$, keeping the other flows as low load traffic.

\subsection{Case-1 (High)}

Since the number of high load traffic increase, it will result in overloaded traffic. So the resulting blocking probability linearly increases whereas the throughput and utilization decreases linearly.

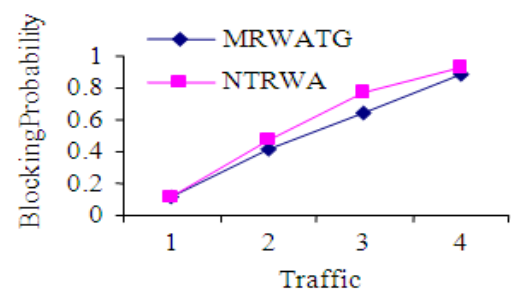

Fig. 5. No. of traffic Vs blocking probability

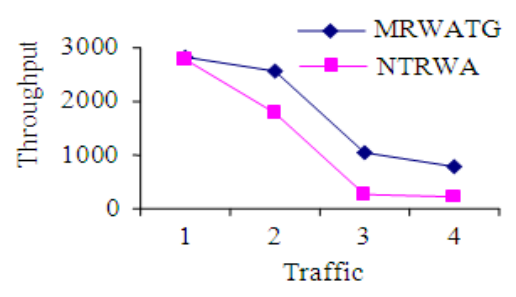

Fig. 6. No. of traffic Vs throughput

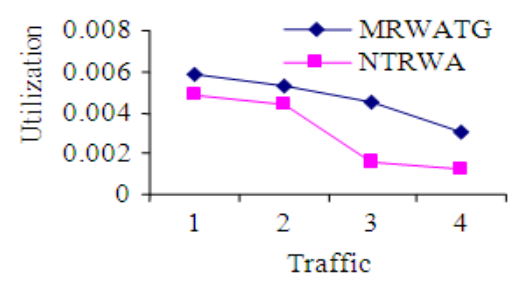

Fig. 7. No. of traffic Vs utilization

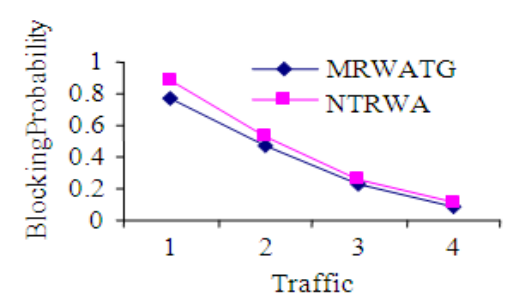

Fig. 8. No. Of Traffic Vs Blocking Probability

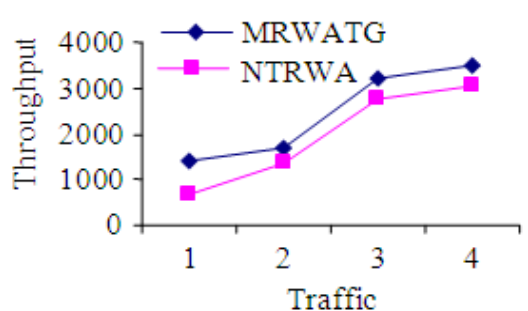

Fig. 9. No. of traffic Vs throughput

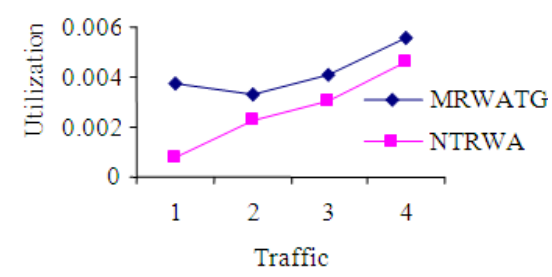

Fig. 10. No. of traffic Vs utilization

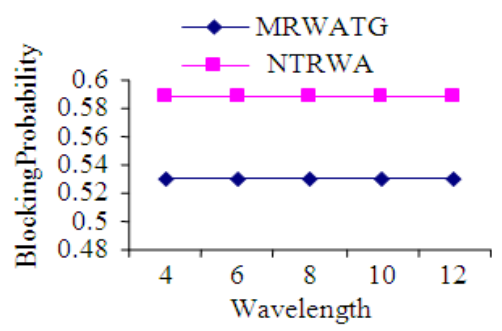

Fig. 11. No of wavelength Vs blocking probability

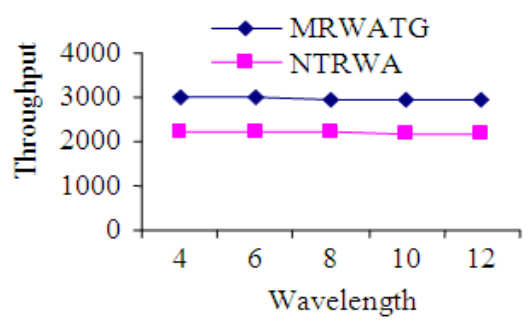

Fig. 12. No of wavelength Vs throughput

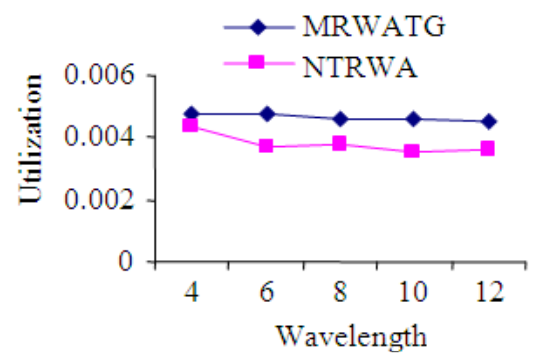

Fig. 13. Wavelength Vs utilization 
We can see this from the Fig. 5-7. From that figure it is evident that blocking probability of proposed MRWATG is slightly less than that of NTRWA and throughput and utilization are more. This is because of the Large Traffic First algorithm of MRWATG.

\subsection{Case2 (Low)}

In the second experiment, we vary the number of low load traffic flows as $1,2 \ldots .4$, keeping the other flows as high load traffic.

Since the number of low load traffic increase, the number of high load traffic will decrease resulting in less number of rejected requests. So the blocking probability linearly decreases whereas the throughput and utilization increases linearly. We can see this from the Fig. 8-10. From that figure, it is also evident that blocking probability of proposed MRWATG is slightly less than that of NTRWA and throughput and utilization are more. This is because of the Large Traffic First algorithm of MRWATG.

\subsection{Based on Number of Wavelengths}

In the second experiment, we vary the number of wavelength per link as $4,6,8,10 \ldots 12$ with number of 2 low load and 2 high load traffic flows and measure the throughput and channel utilization.

From Fig. 11, we can see that the Blocking Probability of our proposed MRWATG is less than the Normal method.

From Fig. 12, we can see that the Throughput of our proposed MRWATG is high than the Normal method.

From Fig. 13, we can see that the Channel Utilization of our proposed MRWATG is high than the Normal method.

\section{CONCLUSION}

In this study, we have proposed a traffic grooming algorithm in order to enhance the traffic. Here, Maximizing Single-Hop Traffic (MSHT-PAVTD) algorithm is used to establish light paths between node pairs having the largest amounts of traffic. Among the established light paths, the risk-disjoint paths are obtained using the Large Traffic First algorithm (LTF-SWCSR). The link weights of the network are updated according to the current network state. Here dijkstra algorithm is used to establish lightpath for shared protection and two times Dijkstra's risk-disjoint path algorithm are used for establishing lightpaths for dedicated protection. After establishing lightpaths, we propose a technique in which router placed at the source which transfers the traffic to the connection node and then the connection node transfers it to the destination. The router calculates the overloaded traffic at the congested node as the difference between the total traffic and the traffic handled. The wavelength allocation is processed based upon the ILP technique and selects cost function as the main criteria. From simulation results we show that this algorithm involves additional capacity in the virtual topology to support survivable routing of connections.

\section{REFERENCES}

Ab-Rahman, M.S., 2012. Flexible topology migration in optical cross add and drop multiplexer in metropolitan ring network. J. Comput. Sci., 8: 474481. DOI: $10.3844 /$ jessp.2012.474.481

Beshir, A., F. Kuipers, A. Orda and P.V. Mieghem, 2011. Survivable impairment-aware traffic grooming in WDM rings. Proceedings of the 23rd International Teletraffic Congress, Sept. 6-9, IEEE Xplore Press, San Francisco, pp: 158-165.

Chaiwong, K., P. Phromsuphorn, J. Koseeyaporn and P. Wardkein, 2008. A new technique for routing and wavelength assignment for WDM mesh networks. Proceedings of the 11th IEEE Singapore International Conference on Communication Systems, Nov. 19-21, IEEE Xplore Press, Guangzhou, pp: 704-708. DOI: 10.1109/ICCS.2008.4737277

Chen, B., R. Dutta and G.N. Rouskas, 2010. Clustering methods for hierarchical traffic grooming in large-scale mesh WDM networks. J. Optical Commun. Network., 2: 502-514. DOI: 10.1364/JOCN.2.000502

Huang, S. and B. Mukherjee, 2010. Survivable multipath traffic grooming in telecom mesh networks with inverse multiplexing. Optical Commun. Network., J., 2: 545-557. DOI: 10.1364/JOCN.2.000545

Huang, S., M. Xia, C. Martel and B. Mukherjee, 2010. Survivable multipath traffic grooming in telecom mesh networks with inverse multiplexing. J. Optical Commun. Network., 2: 545-557. DOI: 10.1364/JOCN.2.000545

Patel, A.N., C. Gao, J.P. Jue, X. Wang and Q. Zhang et al., 2010. Traffic grooming and regenerator placement in impairment-aware optical WDM networks. Proceedings of the 14th Conference on Optical Network Design and Modeling, (ONDM' 10), IEEE Press Piscataway, NJ, USA., pp: 72-77.

Paul, P. and S. Shrivastava, 2010. Traffic grooming in WDM network using ILP. Int. J. Comput. Appli., 4: 1-8.

Ramesh, G. and S.S. Vadivelu, 2010. Fault localization and alarm correlation in optical WDM networks. Int. J. Inform. Commun. Eng., 6: 108-113.

Ramesh, T.K., S. Ashok, K.B. Bithil, D. Nayanar and P.R. Vaya, 2011. Distributed traffic grooming multipath routing algorithm for all optical WDM networks. Eur. J. Sci. Res., 57: 305-313.

Saleh, A.S. and A.E. Kamal, 2009. Many-to-many traffic grooming in WDM networks. J. Optical Commun. Network., 1: 376-391. DOI: 10.1364/JOCN.1.000376

Sinha, M.K., A.S. Pandey, S.K. Sinha and A. Kumar, 2012. Hybrid nodal architecture for traffic Grooming in WDM networks. Int. J. Emerg. Technol. Adv. Eng., 2: 185-194.

Wang, Y. and B. Ramamurthy, 2004. Survivable traffic grooming with path protection at the connection level in WDM mesh networks. Proceedings of the 1st International Conference on Broadband Networks, Oct. 25-29, IEEE Xplore Press, pp: 310 319. DOI: 10.1109/BROADNETS.2004.80 